\title{
Autoinformes de sintomatología depresiva en drogodependientes: nivel de coincidencia del BDI, SCL-90-R y MCMI-II. ¿Depresión o malestar inespecífico?
}

\author{
Eduardo J. Pedrero Pérez*; Ana López-Durán** \\ *Psicólogo. C.A.D. 4. Ayuntamiento de Madrid. \\ **Psicóloga. Dpto. Psicología Clínica y Psicobiología. Universidad de Santiago de Compostela \\ Enviar correspondencia a: \\ Eduardo J. Pedrero. C/ Bergantín, 11 5․ 28042 - Madrid. Correo-e: ejpedrero@yahoo.es
}

Recibido: 11 de octubre de 2004 Aceptado: 21 de Junio de 2005.

\section{RESUMEN}

Consideramos la necesidad de profundizar en el conocimiento de los instrumentos psicométricos en la evaluación psicopatológica de drogodependientes. Nos centramos en la medida de la depresión y estudiamos tres cuestionarios ampliamente utilizados.

OBJETIVO: Estudiar la coincidencia de SCL-90-R, BDI y MCMI-II en la estimación de dimensiones depresivas en usuarios de drogas en tratamiento, y la idoneidad comparada entre ellos para su uso en la clínica.

MÉTODO: Administramos el SCL-90-R a 344 sujetos en tratamiento por abuso/dependencia de sustancias; 99 cumplimentan también el BDI, 64 el MCMI-II y 181 los tres cuestionarios.

RESULTADOS: Hay diferencias entre las escalas que miden síntomas (SCL-90-R y BDI) y las que estiman la probabilidad de que tales síntomas configuren un síndrome (MCMI-II). El MCMI-II muestra una baja sensibilidad, pero aporta mayor especificidad y estabilidad en las medidas. Todas las escalas del SCL-90-R correlacionan fuertemente con casi todas las escalas sindrómicas del MCMI-II y no sólo con las de Distimia y Depresión Mayor. Las fuertes correlaciones del BDI con todas las escalas del SCL-90-R, especialmente con el Índice General Sintomático, y con casi todas las escalas sindrómicas del MCMI-II, apoyan la idea, propuesta por anteriores estudios, de que estos cuestionarios de síntomas evalúan una dimensión inespecífica de malestar, lo que limita seriamente su utilidad diagnóstica.

CONCLUSIONES: Razonamos la utilidad del uso complementario de ambos métodos: los que exploran síntomas parecen más útiles para el trabajo directo con el paciente, pero ineficaces para tareas diagnósticas.

Palabras-clave: Evaluación, Depresión, SCL-90-R, BDI, MCMIII, Sensibilidad, Adicción.

\section{ABSTRACT}

We considered the need for in-depth knowledge of psychometric instruments in the psychopathologic evaluation of drug-users. We focused our attention on measuring depression, studying three widely used questionnaires.

OBJECTIVE: To explore the coincidence of SCL-90-R, $\mathrm{BDI}$ and $\mathrm{MCMI}-\mathrm{Il}$ in the estimation of depressive dimensions in drug-users undergoing treatment, in addition to comparing their suitability for clinical use.

METHOD: We administered the SCL-90-R to 344 subjects undergoing treatment for substance abuse or dependence (heroin, cocaine, alcohol and cannabis); 99 of them also completed the BDI, 64 the MCMI-II, and 181 the three questionnaires.

RESULTS: There are differences between the scales that measure symptoms (SCL-90-R and BDI) and those that consider the probability that such symptoms form a syndrome (MCMI-II). The MCMI-II shows a low sensitivity but provides greater specificity and stability in its measurements. All the SCL-90-R scales correlate strongly with almost all the MCMIII syndrome scales, and not only with those for dysthymia and major depression. The strong correlations of the BDI with all the SCL-90-R scales, particularly with the General Symptomatic Index, as well as with all the MCMI-II syndrome scales, support the idea, proposed by previous studies, that these symptoms questionnaires evaluate a non-specific dimension of malaise, which limits seriously their diagnostic utility.

CONCLUSIONS: The complementary use of both methods is appropriate: those that explore symptoms appear to be the most useful in working directly with patients, but are ineffective in diagnostic work.

Keywords: Assessment, Depression, SCL-90-R, BDI, MCMIII, Sensitivity, Addiction. 


\section{INTRODUCCIÓN}

$\mathbf{L}$ a evaluación psicológica se revela, en el momento actual, como una actividad ineludible en el contexto de los programas de tratamiento de consumidores de drogas que lo solicitan. Por una parte, es preciso conocer la función que juega la droga en el conjunto de conductas del sujeto, de modo que el consumo de drogas puede estar relacionado con la psicopatología como factor predisponente, desencadenante, coexistente, consecuente o independiente (Meyer, 1986). Por otra parte, el conocimiento de los trastornos asociados al consumo de sustancias permite una más adecuada asignación de recursos al tratamiento, así como una formulación de objetivos individualizados. Los sujetos que tienen un problema con el consumo de sustancias y otro trastorno en el eje I, requieren una atención específica ya que presentan un mayor deterioro, peor pronóstico y la existencia de ambos cuadros implica que uno va a influir en el otro, por lo que el tratamiento debe contemplar ambos (Ochoa, 2000; San, 2004; Ziedonis, 1992).

De entre todos los posibles trastornos, los que están asociados a los estados de ánimo depresivos son los más frecuentes (Regier, Farmer, Rae, Locke, Keith, Judd et al., 1990) y también los que presentan mayores dificultades metodológicas para su estudio (Arias, 2001) en la medida en que, en muchos casos, no se trata de verdaderas entidades mórbidas, sino epifenómenos de la suspensión del consumo (Ochoa, 2000), reacciones frente a estresores crónicos asociados al estilo de vida adictivo (Pérez de los Cobos y Casas, 1993) o reacciones frente a situaciones agudas de pérdida de recursos que favorecen la búsqueda de tratamiento (Pedrero, Puerta, Segura y Martínez-Osorio, 2003).

Se han encontrado elevadas tasas de prevalencia de trastornos depresivos en consumidores de cocaína (Gawin y Kleber, 1986; Sánchez Hervás, Tomás y Morales, 2001), de heroína (Arias, López-lbor y Ochoa, 1997; Kidorf, Disney, King, Neufeld, Beilenson y Brooner, 2004; Martínez Higueras, 1993), de alcohol (Casas y Guardia, 2002; Miller, Klamen, Hoffmann, Norman y Flaherty, 1996), de tabaco (Anda, Williamson, Escobedo, Mast, Giovino y Remington, 1990; Breslau, 1993), de éxtasis (Morgan, 2000; Rubio, Bango, Fadón, Pascual y Santo-Domingo, 1998) y de cualquier otra sustancia adictiva. Sin embargo, las discrepancias en cuanto a la magnitud de tal prevalencia son enormes, oscilando, en los estudios citados entre el 5 y el 60\% (Hesselbrock, Hesselbrock, Tennen, Meyer y Workman,1983); incluso algún artículo señala una prevalencia de sintomatología depresiva del $98 \%$ en sujetos alcohólicos (Soler-Insa, Gascón y Grau, 1992). Las dificultades diagnósticas se asientan fundamentalmente en problemas de índole metodológica: diferencias en las muestras utilizadas (mayor prevalencia en dis- positivos psiquiátricos que en recursos ambulatorios específicos), carácter retrospectivo o prospectivo (los primeros informan consistentemente de mayor prevalencia que los segundos), distintos métodos de evaluación (uso de cuestionarios, entrevistas estructuradas o no, mera impresión clínica), sesgos del investigador (adscripción al modelo de enfermedad o análisis funcional de la conducta), diversidad en el establecimiento de definiciones diagnósticas (más o menos restrictivas), diferencias geográficas, variables sociales, etc. (Ortiz, 1998).

La mayor parte de los trabajos encontrados utilizan los sistemas de diagnóstico categorial (más frecuentemente el DSM III y las versiones siguientes) y los instrumentos desarrollados para el ajuste a las categorías diagnósticas: SADS, DIS, SCID, PRISM, SICD-SAC, etc. (Rubio, 1998), pero en la práctica psicológica es más frecuente el uso de cuestionarios autoadministrados, que admiten la propuesta de Beck (1976) según la cual la depresión es una dimensión continua de modo que cualquier persona puede ser situada en un punto determinado de la misma. Ambos métodos presentan ventajas e inconvenientes, pero se admite en la actualidad que lo deseable es una combinación de ambos, lo que maximiza la posibilidad de incrementar la validez y fiabilidad de los diagnósticos.

En general, las ventajas de un método se corresponden con debilidades del otro y viceversa. Si los cuestionarios representan una forma cómoda y rápida de obtener información, las entrevistas estructuradas requieren una inversión de tiempo que los servicios de atención a drogodependientes rara vez pueden proporcionar. Si los cuestionarios están a merced de los sesgos del paciente las entrevistas están en igual medida sujetos a los sesgos del entrevistador; en consecuencia, la alta fiabilidad de los autoinformes estandarizados se corresponde con una muy baja fiabilidad de las entrevistas, que se agudiza en la medida en que rara vez son aplicadas con las garantías que los estudios previos exigen. Si las entrevistas seleccionan la información atendiendo directamente a los criterios clasificatorios de las categorías diagnósticas, los cuestionarios obtienen una información que no necesariamente encamina a tales criterios, sino que, en muchas ocasiones proporciona una visión general que requiere ser interpretada, lo que frecuentemente nos lleva a preguntarnos qué evalúan realmente. Para solucionar estos problemas, algunas entrevistas se preceden de un cuestionario autoinformado (como la SCID) y la mayor parte de los cuestionarios se someten a estudios de validación para estimar los puntos de corte a partir de los cuales es probable que se cumplan los criterios para estimar un diagnóstico determinado.

Aunque son muchos los cuestionarios que se han elaborado y validado para estudiar directa o indirectamente la sintomatología depresiva, en el presente 
trabajo destacamos tres: el BDI (Beck Depression Inventory, Beck, Ward, Mendelson, Mock, y Erbaugh 1961), el MCMI-II (Millon Clinical Multiaxial Inventory, Millon, 1987) y el SCL-90-R (Derogatis, 1983).

El BDI evalúa la presencia e intensidad de la sintomatología depresiva, a partir de la teoría cognitiva de Beck. Es el autoinforme más utilizado internacionalmente para cuantificar los síntomas depresivos en poblaciones clínicas y normales. La versión española del original consta de 19 items de elección múltiple (Conde, Esteban y Useros, 1976), aunque existe una versión revisada por los autores (Beck, Rush, Shaw y Emery, 1979) que también ha sido validada en población española (Sanz y Vázquez, 1998) y muchos estudios no hacen referencia a la versión utilizada, lo que dificulta la comparabilidad de los datos. Además, existe también una versión española del BDI-II (Sanz, Navarro y Vázquez, 2003; Sanz, Perdigón y Vázquez, 2003).

El SCL-90-R es uno de los instrumentos más utilizados para el autoinforme de síntomas. La versión inicial, el SCL-90 (Symptom Check List-90), fue desarrollada por Derogatis y colaboradores en 1973 a partir del Hopkins Symptom Check List (Parloff, Kelman y Frank, 1954). Con posterioridad se presentó la versión revisada de aquél, que fue adaptada al castellano por González de Rivera y cols. (Derogatis, 2002). Se trata de un cuestionario autoadministrado compuesto de 90 items, valorados cada uno en una escala de Likert de 0 (nada) a 4 (mucho), en relación a la magnitud en que la persona se ha sentido molesta por cada uno de los síntomas durante la última semana. Los 90 items proporcionan información en relación a nueve dimensiones sintomáticas (somatización, obsesión/compulsión, sensibilidad interpersonal, depresión, ansiedad, hostilidad, ansiedad fóbica, ideación paranoide, psicoticismo), así como a tres escalas generales: índice general sintomático (o de gravedad), total de síntomas positivos e índice de malestar. Se dispone también de una versión reducida, el Brief Symptom Inventory (Derogatis, 1975) que reduce de 90 a 53 los items y presenta una buena correlación en todas las escalas (por encima de 0,90) con el SCL-90-R.

El Inventario Clínico Muitiaxial de Millon II (MCM-II) cuenta con una adaptación española (Millon, 1999). Es un instrumento concebido para explorar los trastornos de la personalidad desde una perspectiva dimensional que, además, ha sido adaptado para proporcionar una adecuación a los modelos categoriales. Su sistema clasificatorio de referencia es el DSM-III-R, aunque ya está disponible la versión MCMI-III que lo hace a partir del DSM-IV, si bien no disponemos aún de estudios de validez en población española. Consta de 175 items de respuesta verdadero-falso que informan sobre 8 patrones clínicos de personalidad, 3 formas graves de patología de personalidad, 6 síndromes clínicos de intensidad moderada (entre los que se encuentra la escala D de Distimia) y 3 síndromes clínicos graves (entre ellos la escala CC de Depresión Mayor). Las puntuaciones directas, por una parte, permiten la descripción dimensional de los diversos patrones desadaptativos y, por otra, se convierten en puntuaciones de tasa base atendiendo a la distribución de la prevalencia de cada trastorno en los subgrupos de población. La lógica de esta transformación se sitúa en el hecho de que, a diferencia de los rasgos, que se distribuyen normalmente y con frecuencias comparables, los trastornos de personalidad no presentan una distribución semejante ni su prevalencia es igual entre las poblaciones de pacientes, lo que obliga a transformar las puntuaciones atendiendo al modelo de prevalencia subyacente a cada trastorno. Una vez considerado éste, mediante estudios poblacionales preliminares, el autor elabora unas tablas para cada subgrupo de población, estimando un punto de corte en el valor 75, siendo los valores iguales o superiores sugestivos de algún problema clínico, y otro en el valor 85 que indicaría una severidad importante del trastorno. El cuestionario cuenta con diversas medidas de validez, deseabilidad y sinceridad.

Como puede observarse, existen diferencias entre los tres cuestionarios en relación a qué pretenden medir: si el SCL-90-R mide síntomas, algunos de los cuales se agrupan en la escala de Depresión, el BDI explora directa y excluyentemente la sintomatología depresiva y el MCMI-II obtiene una probabilidad de que los síntomas manifestados puedan subsumirse en un cuadro sindrómico.

Encontramos diversos estudios que evalúan la relación entre estos autoinformes. Gotlib y Cane (1989) realizan una revisión sobre los cuestionarios que evalúan depresión y ansiedad, estableciendo correlaciones entre los mismos. En el caso del BDI y el SCL-90-R, recogen que el primero correlaciona significativamente con todas las escalas de síntomas del segundo, y aunque la mayor correlación es con la escala de depresión $(0,70)$, la correlación con la escala de ansiedad también es muy alta (0,67). Brophy, Norvell y Kiluk (1988) señalan al comparar también ambos cuestionarios que todas las dimensiones del SCL-90-R miden constructos similares y que por lo tanto evalúa una dimensión general de psicopatología, más que la presencia de síntomas específicos. Moffett y Radenhausen (1990) evalúan la presencia de síntomas de depresión en sujetos con problemas con el consumo de sustancias en tratamiento. Administran el BDI y la escala de depresión del SCL-90-R en dos ocasiones, encontrando correlaciones de 0,82 y de 0,90. Wetzler, Kahn, Strauman y Dubro (1989) evalúan, en un grupo de pacientes con diagnóstico de depresión mayor, la utilidad de las subescalas de depresión del MCMI, SCL-90-R y del MMPI y la posibilidad de establecer un punto de corte para dicho trastorno. Indican que el punto de corte en el caso del MCMI es de 85 y en el SCL-90-R es de T=70 en la 
escala de población normal. Strauman y Wetzler (1992) analizan la estructura factorial combinada de SCL-90 y MCMI, llegando a la conclusión de que la interpretación por separado de ambos instrumentos proporciona poca información, pero que la factorialización ofrece dos factores, depresión ansiosa/emocionalidad y pensamiento paranoide, que permiten asignar los resultados a una determinada configuración de síntomas. Por último, Gotlib y Cane (1989) concluyen que las diferencias entre los distintos cuestionarios que evalúan depresión radica en el porcentaje de ítems de cada una de las escalas que realmente evalúan depresión y no otros constructos como la ansiedad. En el caso del BDI, el $76 \%$ de los ítems evalúan síntomas de depresión; en la subescala de depresión del SCL-90-R, el 69\%; y en la escala de distimia del MCMI sólo el $47 \%$ de los ítems evalúan depresión.

El SCL-90-R ha mostrado en diversos estudios una elevada sensibilidad diagnóstica, entre el 80 y el $90 \%$, pero una muy baja especificidad, entre el 20 y el $60 \%$ (Aben, Verhey, Lousberg, Lodder y Honig, 2002, Franken y Hendricks, 2001). El BDI mejora estas cifras, llegando a superar el 75\% por ciento de especificidad para la depresión (Richter, Werner, Heerlein, Kraus y Sauer, 1989; Weiss y Griffin, 1989). El manual del MCMI-II (Millon, 1999) propone una sensibilidad del $81 \%$ y una especificidad del $83 \%$ para la escala de Distimia, siendo estas cifras respectivamente del 68 y el $96 \%$ para la Depresión Mayor. Otros estudios no son tan optimistas, encontrando una sensibilidad del $37 \%$ y del $57 \%$, y una especificidad del 41 y del $59 \%$ para los respectivos síndromes, encontrándose que la tercera versión del test no mejora sustancialmente los resultados (Retzlaff, 1996). Finalmente, otros trabajos discrepan de estas cifras, atribuyendo al MCMI-II un $71 \%$ y un $85 \%$, y al SCL-90-R un $68 \%$ y un $69 \%$ en las respectivas escalas de depresión (Marlowe y Wetzler, 1994).

El objetivo de nuestro estudio es evaluar la coincidencia, a la hora de valorar la presencia de sintomatología depresiva, de tres cuestionarios: el BDI, la escala de depresión del SCL-90-R y las escalas de distimia y depresión mayor del MCMI-II, así como discutir sobre la idoneidad de cada uno de ellos, en función de sus características, para la aplicación a personas en tratamiento por abuso/dependencia de sustancias.

\section{MÉTODO}

\section{Participantes}

La muestra está formada por 344 sujetos que están en tratamiento por abuso o dependencia de sustancias en el Centro de Atención a Drogodependencias del Ayuntamiento de Madrid (CAD-4), en las Unidades Asistenciales de Drogodependencias de Pontevedra, Alborada (Vigo) y A.C.L.A.D. (A Coruña), y en las Comunidades Terapéuticas de Alborada (Tomiño) y Fonte Douro (A Coruña).

La muestra final está compuesta por 344 sujetos, 249 varones (72\%) y 95 mujeres (28\%); de ellos, 120 (35\%) realizan tratamiento por abuso/dependencia de heroína, 143 (42\%) por abuso/dependencia de cocaína, $69(20 \%)$ por abuso/dependencia de alcohol y 12 (3\%) por abuso/dependencia de cannabis. La edad de media del grupo es de 33,9 años (d.e. 7,7), con un rango que va de los 16 a los 63 años. Como media llevan 14 años (d.e. 7,4$)$ consumiendo la droga, en cuyo consumo se iniciaron a los 19,7 años (d.e. 5,6). De ellos, 180 se encuentran en el Grupo de Inicio, 34 en el de Preparación, 84 en el de Acción y 46 en el de Mantenimiento (Tabla 1).

\section{Instrumentos}

El SCL-90-R (Derogatis, 2002) es un instrumento que nos permite conocer la presencia de determinados síntomas durante la última semana, incluyendo el día de hoy, sin llegar a establecer un diagnóstico. Una

Tabla 1. Descriptivos de la muestra en variables sociodemográficas y de consumo, según los grupos por droga principal.

\begin{tabular}{|c|c|c|c|c|c|c|c|c|c|c|c|c|c|}
\hline & \multirow[b]{2}{*}{ N } & \multicolumn{2}{|c|}{ Edad } & \multicolumn{2}{|c|}{ Sexo } & \multicolumn{4}{|c|}{ Grupo } & \multicolumn{2}{|c|}{ Tiempo consumo } & \multicolumn{2}{|c|}{ Edad de inicio } \\
\hline & & Media & D.E. & Varón & Mujer & Inicio & Prepar. & Acción & Manten. & Media & D.E. & Media & D.E. \\
\hline HEROÍNA & 120 & 34,7 & 5,9 & 83 & 37 & 59 & 7 & 27 & 27 & 14,3 & 6,4 & 20,3 & 5,3 \\
\hline COCAÍNA & 143 & 30,8 & 7,1 & 108 & 35 & 69 & 23 & 42 & 9 & 11,1 & 6,3 & 19,6 & 5,2 \\
\hline ALCOHOL & 69 & 39,6 & 8,2 & 52 & 17 & 45 & 1 & 15 & 8 & 20,2 & 7,7 & 19,5 & 7,0 \\
\hline CANNABIS & 12 & 28,3 & 7,3 & 6 & 6 & 7 & 3 & 0 & 2 & 12,5 & 6,1 & 15,8 & 2,5 \\
\hline TOTAL & 344 & 33,8 & 7,7 & 249 & 95 & 180 & 34 & 84 & 46 & 14,1 & 7,4 & 19,7 & 5,6 \\
\hline
\end{tabular}


de las subescalas que lo forman es la de depresión, la cual está formada a su vez por 13 ítems. El formato de respuesta es una escala tipo Likert entre cero y cuatro.

El MCMI-II (Millon, 1999) es un cuestionario que consta de 175 ítems. Contiene 10 escalas básicas de personalidad, tres de personalidad patológica, seis síndromes clínicos de gravedad moderada y tres síndromes clínicos graves. Para el estudio utilizaremos la escala de distimia de los síndromes clínicos de gravedad moderada, y la escala de depresión mayor de los síndromes clínicos graves. El formato de respuesta es verdadero-falso.

El BDI (Beck et al., 1979, versión española de Sanz y Vázquez, 1998) evalúa la presencia de síntomas de depresión a través de 21 ítems con formato de respuesta entre cero y tres.

\section{Procedimiento}

Se administran los cuestionarios de forma individual a cada sujeto con la presencia de un psicólogo entrenado para ello, y que en todo momento atiende a las dudas que puedan presentarse. Al menos dos de los tres cuestionarios (el SCL-90-R y otro) son completados en una única sesión, y es a estos, cumplimentados simultáneamente, a los que atendemos en el estudio, descartando el tercero si se ha rellenado en un momento diferente. En todos los casos se advierte a los sujetos que la evaluación que se va a realizar forma parte de su proceso terapéutico y que los datos obtenidos con los cuestionarios pueden ser utilizados en estudios de investigación si no hay objeción por su parte.

Se tiene en cuenta el momento del tratamiento en que se encuentra cada participante, siendo clasificados en los siguientes grupos: 1) Grupo de Inicio, aquellos que cumplimentan el cuestionario en las dos primeras semanas desde la formalización de la demanda de tratamiento; 2) Grupo de Preparación, aquellos que llevan menos de un mes en tratamiento y han acudido al menos a 4 citas de valoración; 3) Grupo de Acción, aquellos que llevan más de un mes en tratamiento; 4) Grupo de Mantenimiento, aquellos que Ilevan al menos tres meses en tratamiento. La evaluación se efectúa siempre que se encuentren estabilizados sin interferencia de sintomatología derivada de la intoxicación o del abandono del consumo.

Finalmente obtenemos una muestra de 181 sujetos que cumplimentan los tres cuestionarios, 99 sólo el SCL-90-R y el BDI y 64 sólo el SCL-90-R y el MCMI-II.

La muestra de 344 sujetos forma parte de una mayor, de la que hemos eliminado a todos los sujetos que presentaban cuestionarios dudosos para su co- rrección, cumplimentados de modo incompleto, o que no cumplían los criterios de validez (un item o más puntuado positivamente en la escala V) del MCMI-II. No hemos aplicado, sin embargo, la norma, propuesta por el autor, de dar por inválidos los items que puntuaban por encima de 590 en la escala de Sinceridad (X) de este cuestionario, puesto, que como presentaremos en los resultados y comentaremos en la discusión, los datos apuntan a que una puntuación elevada en esta escala no indica falta de sinceridad, sino severidad de los trastornos, lo que nos lleva a pensar que si se aplica esta limitación, el test excluiría inadecuadamente a los sujetos con un malestar más acusado. Se trata de 14 sujetos que, en base a la observación clínica, y tras repetir en algunos casos el test, obtuvieron puntuaciones similares e impresionaron de patología severa, pero no de falta de sinceridad. Para ello, prolongamos la tabla de corrección propuesta en el manual estimando las puntuaciones que deberían atribuirse a las puntuaciones obtenidas.

\section{Análisis de datos}

Se ha utilizado el paquete estadístico SPSS 12.0 para Windows. Se han realizado medidas de tendencia central y dispersión, correlaciones bivariadas, pruebas no paramétricas de comparación de medias y análisis de regresión.

\section{RESULTADOS}

Respecto a lo que comentamos previamente sobre la escala de sinceridad X del MCMI-II, como puede observarse en la Tabla 2, la escala X correlaciona positiva, fuerte y consistentemente con la práctica totalidad de escalas tanto del MCMI-II como del SCL90-R, lo que nos reafirma en la idea de que mide más bien intensidad que falta de sinceridad.

Otro aspecto que ha sido analizado es cómo evoluciona la puntuación en el SCL-90-R en función del momento en que se realiza la evaluación. En el Gráfico 1 observamos las puntuaciones medias obtenidas por los diferentes grupos según el momento de tratamiento en que se encuentren, en las escalas sintomatológicas del SCL-90-R, y las comparamos con las que obtiene la población general en el trabajo de validación del cuestionario en España. Se puede comprobar cómo el malestar declarado es máximo al inicio de tratamiento y disminuye a medida que éste avanza, salvo en la escala de Ansiedad Fóbica, que siempre es puntuada como menor por los diferentes grupos de consumidores en tratamiento. 
Tabla 2. Correlaciones ( $r$ de Spearman) entre las escalas de control del MCMI-II y las escalas de síndromes del Eje I del mismo cuestionario Il y algunas escalas y los índices generales del SCL-90$R$, y el BDI en puntuaciones directas. (Se eliminan las correlaciones $p>0,001$ ).

\begin{tabular}{lll|}
\hline & TBX & TBY \\
\hline MCMI-II & & \\
TB ANSIEDAD & 0,50 & \\
TB SOMATOMORFOS & 0,45 & 0,39 \\
TB HIPOMANÍA & 0,58 & \\
TB DISTIMIA & 0,55 & \\
TB ABUSO ALCOHOL & 0,65 & \\
TB ABUSO DROGAS & 0,74 & \\
TB PENSAMIENTO PSICÓTICO & 0,71 & $-0,22$ \\
TB DEPRESIÓN MAYOR & 0,64 & 0,48 \\
TB TRASTORNO DELIRANTE & 0,50 & \\
SCL-9O-R & & \\
IDEACIÓN PARANOIDE & 0,57 & \\
PSICOTICISMO & 0,55 & \\
ÍNDICE GENERAL SINTOMÁTICO & 0,57 & \\
TOTAL DE SÍNTOMAS POSITIVOS & 0,59 & $-0,28$ \\
ÍNDICE DE MALESTAR & 0,46 & \\
BDI & 0,43 & \\
\hline
\end{tabular}

Gráfico 1. Representación gráfica de las puntuaciones medias obtenidas por cada grupo, en función del momento de tratamiento, en las escalas sintomatológicas del SCL-90-R, comparadas por las obtenidas en población general.

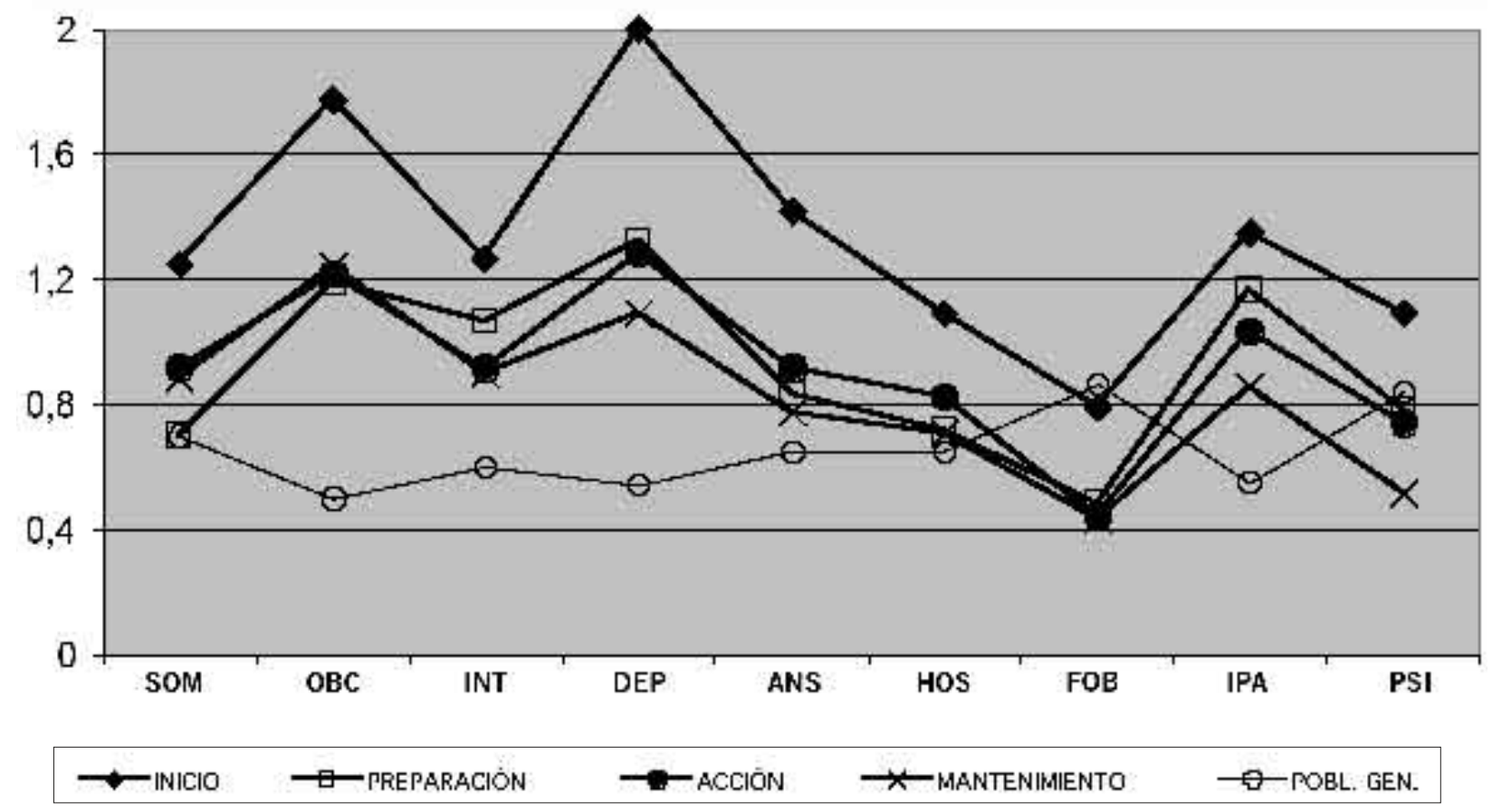

SOM=Somatización, $\quad \mathrm{OBC}=$ Obsesión/Compulsión, INT=Sensibilidad Interpersonal, DEP=Depresión, ANS=Ansiedad, HOS = Hostilidad, FOB=Ansiedad Fóbica, IPA=Ideación Paranoide, PSI=Psicoticismo; POBL.GEN=Población General. Grupo de Inicio $(n=181)$, Preparación $(n=34)$, Acción $(n=84)$ y Mantenimiento $(n=46)$. 
Dentro del grupo que inicia tratamiento, comparamos el malestar declarado por los subgrupos en función de la droga que motiva la demanda (Prueba de Kruskal-Wallis; post-hoc prueba de Tamhane, $p<0,01$ ). Los consumidores de heroína son quienes mayor intensidad de síntomas declaran, a costa, sobre todo, de la sintomatología depresiva, significativamente mayor que la de los consumidores de cocaína, y de la somatización, significativamente mayor que la de consumidores de cocaína y cannabis, siendo estos últimos quienes menor magnitud de síntomas declaran. En la Tabla 3 observamos las diferencias y su significación en los índices generales.

Tabla 3. Puntuaciones medias y desviaciones estándar de los diferentes grupos de sujetos, según la droga principal, en los índices generales del SCL-90-R, y su nivel de significación obtenido mediante la Prueba de Kruskal-Wallis. (Grupo de Inicio, N=180).

\begin{tabular}{|lcccccc|}
\hline SCL-90-R & DROGA & N & Media & D.E. & Chi-cuadrado & Sig. asintót. \\
\hline ÍNDICE GENERAL & HEROÍNA & 59 & 1,56 & 0,7 & 8,38 & 0,04 \\
SINTOMÁTICO & COCAÍNA & 69 & 1,43 & 0,8 & & \\
& ALCOHOL & 45 & 1,21 & 0,7 & & \\
& CANNABIS & 7 & 0,96 & 0,6 & & \\
TOTAL DE SÍNTOMAS & HEROÍNA & 59 & 58,05 & 17,9 & 7,41 & 0,06 \\
POSITIVOS & COCAÍNA & 69 & 52,94 & 21,3 & & \\
& ALCOHOL & 45 & 48,69 & 20,3 & & \\
& CANNABIS & 7 & 43,43 & 21,9 & & 0,06 \\
ÍNDICE DE MALESTAR & HEROÍNA & 59 & 2,32 & 0,6 & 7,31 & \\
& COCAÍNA & 69 & 2,24 & 0,6 & & \\
& ALCOHOL & 45 & 2,06 & 0,6 & & \\
& CANNABIS & 7 & 1,81 & 0,5 & & \\
\hline
\end{tabular}

Se presentan ahora (Gráfico 2) las puntuaciones medias obtenidas por cada grupo, según el momento de tratamiento, en el BDI. En la Tabla 4 desglosamos estos resultados en función de la droga principal; puede observarse cómo la significación obtenida (Prueba de Kruskal-Wallis) es pobre y variable, debido a la desigual composición de los grupos, pero los datos apun- tan a que los consumidores de heroína puntúan consistentemente más alto y los consumidores de cannabis consistentemente más bajo, mientras las puntuaciones de los consumidores de cocaína y alcohol son muy similares en todos los momentos. Las mujeres puntúan sistemáticamente más alto que los varones $\left(\mathrm{M}_{\mathrm{v}}=15,3\right.$; $M_{m}=18,6 ; N_{v}=202 ; N_{m}=78$ : Sig. asintót. bil=0,015).

Tabla 4. Valores medios y desviaciones estándar de las puntuaciones en el BDI de los subgrupos en función de la droga principal y el momento de tratamiento; significación de las diferencias (Prueba de Kruskal-Wallis).

\begin{tabular}{|c|c|c|c|c|c|c|c|c|}
\hline & \multicolumn{2}{|r|}{ INICIO } & \multicolumn{2}{|c|}{ PREPARACIÓN } & \multicolumn{2}{|c|}{ ACCIÓN } & \multicolumn{2}{|c|}{ MANTENIMIENTO } \\
\hline & $\mathrm{N}$ & M (D.E.) & $N$ & $M(D . E)$. & $\mathrm{N}$ & M (D.E.) & $N$ & $\mathrm{M}(D . E)$. \\
\hline HEROÍNA & 57 & $22,3(8,7)$ & 3 & $26,7(3,8)$ & 14 & $16,9(6,6)$ & 23 & $12,4(9,8)$ \\
\hline COCAÍNA & 53 & $17,7(8,2)$ & 22 & $13,2(8,9)$ & 37 & $11,0(6,9)$ & 8 & $9,3(8,0)$ \\
\hline ALCOHOL & 39 & $17,6(9,2)$ & 1 & $23,0(-)$ & 6 & $11,5(5,7)$ & 7 & $8,4(7,1)$ \\
\hline CANNABIS & 6 & $15,0(8,5)$ & 2 & $10,0(14,1)$ & 0 & - & 2 & $3,0(4,2)$ \\
\hline Total & 155 & $19,2(8,9)$ & 28 & $14,8(9,6)$ & 57 & $12,5(7,1)$ & 40 & $10,6(9,0)$ \\
\hline Sig. & & 0,02 & & 0,11 & & 0,03 & & 0,31 \\
\hline
\end{tabular}


Gráfico 2. Puntuaciones medias obtenidas por cada grupo en el BDI en función del momento de tratamiento.

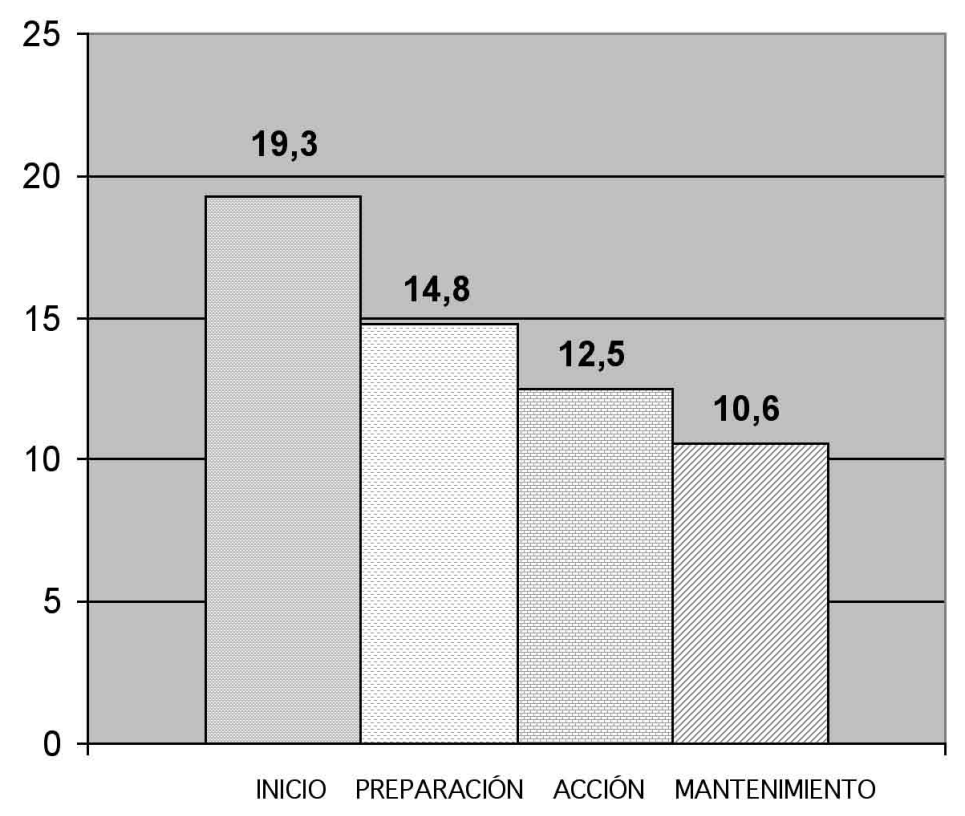

Al atender a la distribución de puntuaciones TB medias, según el momento de tratamiento, en las escalas sindrómicas del MCMI-II, podemos observar que, salvo en la escala de Ansiedad $(p<0,01)$ y en la de Depresión Mayor $(p<0,05)$, las diferencias no son significativas. En el Gráfico 3 se presentan los valores medios en las mismas escalas en el grupo que inicia tratamiento, según la droga principal, observándose cómo las curvas prácticamente se superponen, salvo en el caso de los consumidores de cannabis que puntúan sensiblemente más bajo, si bien tal diferencia es sólo aparente y carece de significación estadística al presentar ese grupo un número tan escaso de miembros.

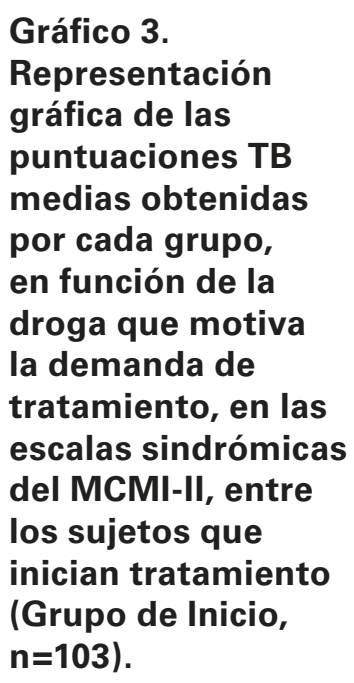

Gráfico 3.

Representación gráfica de las puntuaciones TB medias obtenidas por cada grupo, en función de la droga que motiva la demanda de tratamiento, en las escalas sindrómicas del MCMI-II, entre los sujetos que inician tratamiento $n=103$ ).

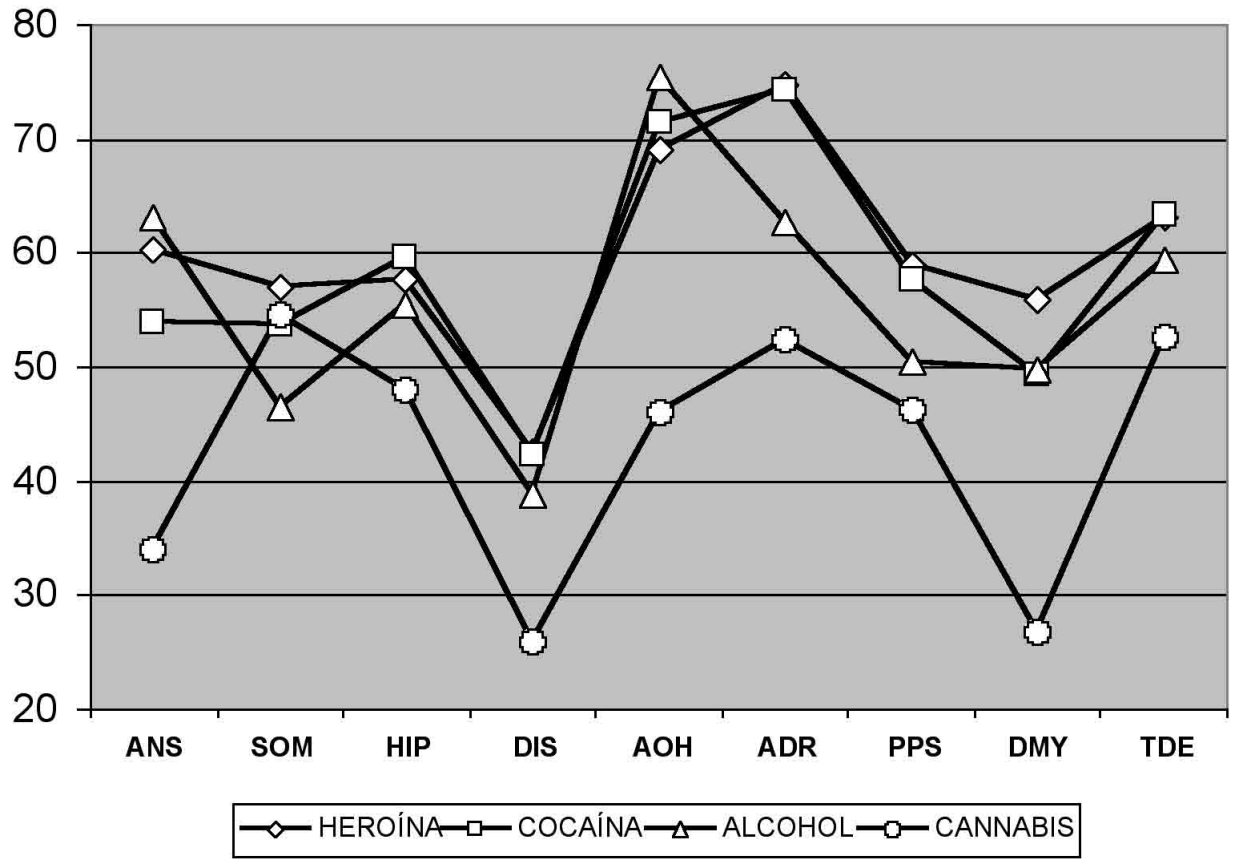

ANS =Ansiedad, SOM=Somatomorfos, HIP=Hipomanía, DIS=Distimia, AOH=Abuso de Alcohol, ADR=Abuso de Drogas, PPS = Pensamiento Psicótico, DMY=Depresión Mayor, TDE=Trastorno Delirante. (Heroína N= 29; Cocaína N=45; Alcohol N= 23; Cannabis $\mathrm{N}=6$ ) 
Sí aparecen diferencias significativas en varias escalas cuando hacemos la comparación por sexos, puntuando, en el Grupo de Inicio $(\mathrm{N}=103)$, más alto las mujeres en Hipomanía (U de Mann-Whitney, p>0,01), Somatomorfos, Distimia, Abuso de Drogas y Depresión Mayor $(p<0,05)$, y más bajo en Abuso de Alcohol $(p<0,01)$, diferencias consistentes en el resto de momentos de tratamiento, aunque con diversa significación.

Se atiende ahora a las escalas B (Abuso de Alcohol) y T (Abuso de Drogas) del MCMI-II. Este cuestionario establece la puntuación TB $=75$ como punto crítico para determinar la probabilidad de un diagnóstico de abuso o dependencia. Sin embargo, comprobamos cómo únicamente el $61 \%$ de los sujetos diagnosticados de abuso o dependencia de alcohol, que inician tratamiento, alcanzan esa puntuación criterio, porcentaje que corresponde, en la escala $\mathrm{T}$, al $48 \%$ de los consumidores de heroína, $47 \%$ de cocaína y sólo un 17\% de los consumidores de cannabis.

Procedemos ahora a estudiar las relaciones observadas entre las medidas obtenidas por los tres cuestionarios. La Tabla 6 muestra las correlaciones que presentan entre sí los tres cuestionarios. La Tabla 7 se centra en las escalas que miden depresión en los tres cuestionarios, pero utilizamos, en esta ocasión, las puntuaciones TB del MCMI-II, puesto que éstas ya contienen las correcciones propuestas por el autor para ajustar a la prevalencia estimada.

Tabla 5. Porcentaje de sujetos del Grupo de Inicio de tratamiento $(N=103)$ que puntúan por debajo y por encima del punto de corte $T B=75$ en las escalas $B$ (Abuso de alcohol) y $T$ (Abuso de drogas) en el MCMI-II.

\begin{tabular}{|lccccc|}
\hline & & \multicolumn{4}{c|}{ DROGA PRINCIPAL } \\
\cline { 3 - 6 } & & HEROÍNA & COCAÍNA & ALCOHOL & CANNABIS \\
\hline \multirow{2}{*}{ Escala B } & TB $<75$ & $62,1 \%$ & $60,0 \%$ & $39,1 \%$ & $100,0 \%$ \\
& TB $>74$ & $37,9 \%$ & $40,0 \%$ & $\mathbf{6 0 , 9 \%}$ & $0,0 \%$ \\
\hline \multirow{2}{*}{ Escala T } & TB $<75$ & $51,7 \%$ & $53,3 \%$ & $69,6 \%$ & $83,3 \%$ \\
& TB $>74$ & $\mathbf{4 8 , 3} \%$ & $\mathbf{4 6 , 7 \%}$ & $30,4 \%$ & $\mathbf{1 6 , 7 \%}$ \\
\hline
\end{tabular}

Tabla 6. Correlaciones no paramétricas ( $r$ de Spearman) entre las escalas e índices generales del SCL-90, las escalas de síndromes clínicos del MCMI-II y el BDI, en puntuaciones directas. (Se han eliminado las correlaciones significativas para $p>0,001$ ).

\begin{tabular}{|c|c|c|c|c|c|c|c|c|c|c|}
\hline & Ansiedad & Somatomorfos & Hipomanía & Distimia & $\begin{array}{l}\text { Abuso } \\
\text { alcohol }\end{array}$ & $\begin{array}{l}\text { Abuso } \\
\text { drogas }\end{array}$ & $\begin{array}{l}\text { Pensam. } \\
\text { psicótico }\end{array}$ & $\begin{array}{l}\text { Depres. } \\
\text { mayor }\end{array}$ & $\begin{array}{l}\text { Transt. } \\
\text { delirante }\end{array}$ & BDI \\
\hline SOMATIZACIÓN & 0,56 & 0,47 & & 0,45 & 0,34 & 0,30 & 0,43 & 0,53 & & 0,61 \\
\hline $\begin{array}{l}\text { OBSESIÓN/ } \\
\text { COMPULSIÓN }\end{array}$ & 0,59 & 0,45 & & 0,53 & 0,46 & 0,32 & 0,57 & 0,63 & & 0,72 \\
\hline $\begin{array}{l}\text { SENSIBILIDAD } \\
\text { INTERPERSONAL }\end{array}$ & 0,41 & 0,33 & & 0,44 & 0,40 & 0,33 & 0,53 & 0,52 & & 0,65 \\
\hline DEPRESIÓN & 0,61 & 0,47 & & 0,57 & 0,45 & 0,26 & 0,52 & 0,68 & & 0,83 \\
\hline ANSIEDAD & 0,64 & 0,53 & 0,22 & 0,53 & 0,49 & 0,37 & 0,55 & 0,64 & & 0,70 \\
\hline HOSTILIDAD & 0,51 & 0,42 & 0,28 & 0,44 & 0,43 & 0,45 & 0,46 & 0,53 & & 0,53 \\
\hline $\begin{array}{l}\text { ANSIEDAD } \\
\text { FÓBICA }\end{array}$ & 0,50 & 0,39 & & 0,47 & 0,47 & 0,27 & 0,47 & 0,55 & & 0,55 \\
\hline $\begin{array}{l}\text { IDEACIÓN } \\
\text { PARANOIDE }\end{array}$ & 0,41 & 0,34 & 0,27 & 0,38 & 0,42 & 0,40 & 0,60 & 0,47 & 0,31 & 0,56 \\
\hline PSICOTICISMO & 0,53 & 0,44 & 0,20 & 0,53 & 0,49 & 0,37 & 0,65 & 0,61 & & 0,72 \\
\hline $\begin{array}{l}\text { ÍNDICE GENERAL } \\
\text { SINTOMÁTICO }\end{array}$ & 0,64 & 0,52 & 0,24 & 0,58 & 0,51 & 0,39 & 0,62 & 0,69 & & 0,80 \\
\hline $\begin{array}{l}\text { TOTAL DE SÍNTOMAS } \\
\text { POSITIVOS }\end{array}$ & 0,61 & 0,52 & 0,23 & 0,54 & 0,50 & 0,38 & 0,58 & 0,63 & & 0,70 \\
\hline ÍNDICE DE MALESTAR & 0,52 & 0,41 & 0,21 & 0,51 & 0,39 & 0,30 & 0,53 & 0,58 & & 0,73 \\
\hline $\mathrm{BDI}$ & 0,56 & 0,37 & & 0,54 & 0,44 & & 0,49 & 0,65 & & \\
\hline
\end{tabular}


Tabla 7. Correlaciones ( $r$ de Spearman) entre las medidas de depresión: BDI, escala de Depresión del SCL-90-R (ambas en puntuaciones directas) y escalas D (Distimia) y CC (Depresión Mayor) del MCMI-II (en puntuaciones TB). (En negrita significación $p<0,001$ ).

\begin{tabular}{|ll|ccc|}
\cline { 3 - 5 } & & $\begin{array}{c}\text { DEPRESIÓN } \\
\text { SCL-90-R }\end{array}$ & $\begin{array}{c}\text { DISTIMIA } \\
\text { (MCMI-II) }\end{array}$ & $\begin{array}{c}\text { DEPRESIÓN MAYOR } \\
\text { (MCMI-II) }\end{array}$ \\
\hline BDI & $r$ & $\mathbf{0 , 8 3}$ & $\mathbf{0 , 5 4}$ & $\mathbf{0 , 6 5}$ \\
& $N$ & 280 & 181 & 181 \\
\hline \multirow{2}{*}{ DEPRESIÓN SCL-90-R } & $r$ & & $\mathbf{0 , 5 7}$ & $\mathbf{0 , 6 8}$ \\
& $N$ & & 245 & 245 \\
\hline \multirow{2}{*}{ DISTIMIA (MCMI-II) } & $r$ & & & $\mathbf{0 , 7 7}$ \\
& $N$ & & & 245 \\
\hline
\end{tabular}

Se comparan en la Tabla 8 las puntuaciones que obtienen los sujetos en las escalas sintomatológicas del SCL-90-R y el BDI, en función de que alcancen las puntuaciones criterio ( $\mathrm{TB}=75)$ para estimar la presencia de un síndrome de ansiedad, distimia o depresión mayor en el MCMI-II.

En la Tabla 9 podemos observar el modelo de regresión de las escalas sintomatológicas del SCL-90-R sobre el Índice General de Malestar, pudiéndose apreciar que la escala de Ansiedad predice por sí misma un 83\% de la varianza del IGS, y que la escala de Depresión apenas aporta un $9 \%$ más. Si eliminamos la escala de Ansiedad, la de Depresión predice también por sí misma un $83 \%$ de la varianza del ICS. Ambas escalas correlacionan 0,91 con el IGS ( $N=344$ para ambas pruebas)

Tabla 8. Puntuaciones directas medias y desviación estándar en las escalas de Depresión y Ansiedad del SCL-90-R y el BDI, de los sujetos que obtienen puntuaciones TB por encima y por debajo de la puntuación criterio (TB=75) para estimar la presencia de un síndrome en el MCMI-II.

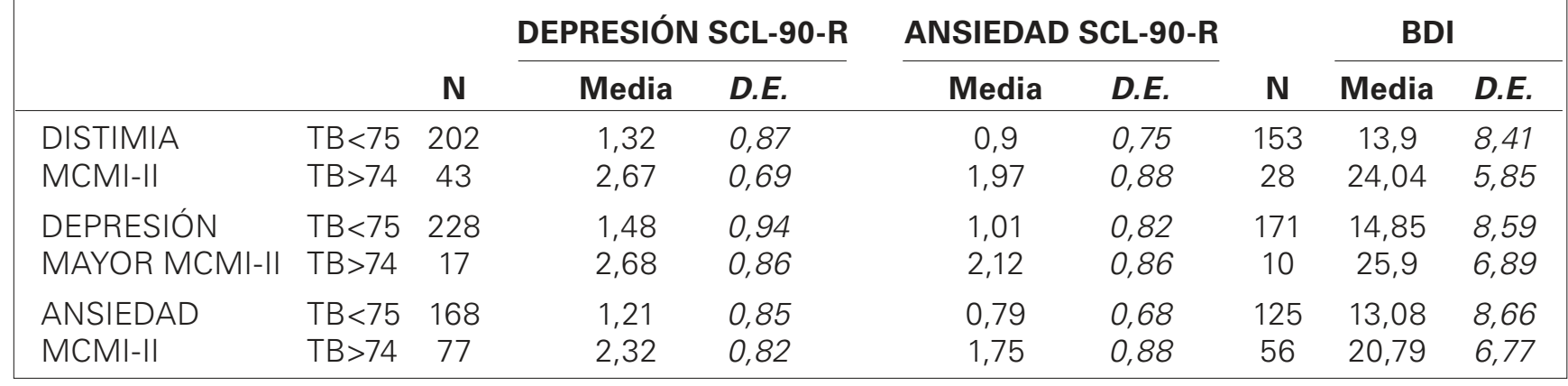

Tabla 9. Resumen del modelo de regresión de las escalas sobre el Índice General Sintomático del SCL-90-R.

\begin{tabular}{lcccc|}
\hline Modelo & $\mathbf{R}$ & $\mathbf{R}^{\mathbf{2}}$ & $\mathbf{R}^{\mathbf{2}}$ corregida & Error típ. de la estimación \\
\hline ANSIEDAD & 0,91 & 0,83 & 0,83 & 0,31 \\
DEPRESIÓN & 0,96 & 0,91 & 0,91 & 0,22 \\
SENSIBILIDAD INTERPERSONAL & 0,97 & 0,94 & 0,94 & 0,18 \\
SOMATIZACIÓN & 0,98 & 0,96 & 0,96 & 0,15 \\
PSICOTICISMO & 0,98 & 0,97 & 0,97 & 0,14 \\
OBSESIÓN/COMPULSIÓN & 0,99 & 0,97 & 0,97 & 0,13 \\
IDEACIÓN PARANOIDE & 0,99 & 0,97 & 0,97 & 0,12 \\
ANSIEDAD FÓBICA & 0,99 & 0,97 & 0,97 & 0,12 \\
HOSTILIDAD & 0,99 & 0,98 & 0,97 & 0,12 \\
\hline
\end{tabular}


La Tabla 10 explora la capacidad predictiva de las dimensiones sintomatológicas del SCL-90-R sobre cada una de las escalas sindrómicas del MCMI-II, mediante un análisis de regresión por pasos sucesivos. Se efectúa la misma operación sobre el BDI, obteniéndose una fórmula de regresión en la cual la puntuación de la escala de Depresión del SCL-90-R predice por sí misma un $68 \%$ de la varianza del BDI, que alcanza al $70 \%$ cuando se tienen en cuenta también las de Psicoticismo, Somatización y Hostilidad (esta última, en negativo).

Tabla 10. Coeficientes beta estandarizados y $\mathbf{R}^{2}$ corregida de la fórmula de regresión de las escalas sintomáticas del SCL-90-R sobre cada una de las escalas sindrómicas del MCMI-II (N=245).

\begin{tabular}{|c|c|c|c|c|c|c|c|c|c|}
\hline & Ansiedad & Somatomorfos & Hipomanía & Distimia & $\begin{array}{l}\text { Abuso } \\
\text { alcohol }\end{array}$ & $\begin{array}{l}\text { Abuso } \\
\text { drogas }\end{array}$ & $\begin{array}{c}\text { Pensamiento } \\
\text { psicótico }\end{array}$ & $\begin{array}{l}\text { Depresión } \\
\text { mayor }\end{array}$ & $\begin{array}{l}\text { Trastorno } \\
\text { delirante }\end{array}$ \\
\hline SOMATIZACIÓN & 0,17 & 0,19 & & & & & & & \\
\hline $\begin{array}{l}\text { OBSESIÓN/ } \\
\text { COMPULSIÓN }\end{array}$ & & 0,21 & & & & & 0,20 & 0,26 & \\
\hline \multicolumn{10}{|l|}{$\begin{array}{l}\text { SENSIBILIDAD } \\
\text { INTERPERSONAL }\end{array}$} \\
\hline DEPRESIÓN & 0,27 & & & 0,39 & & & & 0,44 & $-0,26$ \\
\hline ANSIEDAD & 0,29 & 0,23 & & 0,16 & 0,29 & & & & \\
\hline HOSTILIDAD & & & 0,18 & & & 0,28 & & & \\
\hline \multicolumn{10}{|l|}{$\begin{array}{l}\text { ANSIEDAD } \\
\text { FÓBICA }\end{array}$} \\
\hline $\begin{array}{l}\text { IDEACIÓN } \\
\text { PARANOIDE }\end{array}$ & & & 0,26 & & & & 0,20 & & 0,48 \\
\hline PSICOTICISMO & & & & 0,16 & 0,27 & 0,26 & 0,25 & & \\
\hline$R^{2}$ & 0,44 & 0,3 & 0,15 & 0,4 & 0,27 & 0,22 & 0,32 & 0,44 & 0,14 \\
\hline
\end{tabular}

\section{DISCUSIÓN}

La importancia indiscutible de conocer las variables psicopatológicas que acompañan a la conducta de autoadministración de sustancias nos obliga a elegir los instrumentos que nos informen de forma más exacta y completa de tales condiciones, de cara a formular las estrategias y los objetivos más adecuados en cada caso. En cuanto a los cuestionarios, necesitamos conocer sus propiedades en cuanto a fiabilidad, validez, sensibilidad y especificidad, pero también la facilidad para ser cumplimentados por los pacientes destinatarios y los resultados que, en cuanto a la comparabilidad, nos permitan seleccionar aquellos más breves y que no dupliquen información. Dado que son muchas las cuestiones a evaluar, es preciso delimitar una batería sencilla y apropiada. Esta es la razón que nos mueve a efectuar este estudio.

En concreto, hemos comparado tres instrumentos. Uno (BDI), mide la depresión como entidad independiente y desde una perspectiva dimensional. Otro
(SCL-90-R), mide la depresión también desde un enfoque dimensional, pero relacionada con otras ocho dimensiones sintomatológicas. Finalmente, el tercero (MCMI-II) nos informa de la probabilidad de que esos síntomas puedan configurar un cuadro sindrómico de distimia o depresión mayor.

De los 21 items con los que cuenta el BDI, $17 \mathrm{cu}$ bren 8 de los 9 criterios sintomáticos diagnósticos del episodio depresivo mayor del DSM-IV (Sanz y Vázquez, 1998), si bien ello no justifica en ningún caso el uso exclusivo del BDI como un instrumento diagnóstico de depresión (Vázquez, 1995). En nuestra muestra observamos cómo la puntuación media de todos los sujetos que inician tratamiento se sitúa justo en el punto de corte que los estudios de validación han considerado como indicativo de la existencia de una depresión, mientras que los sujetos que continúan en tratamiento declaran progresivamente menos sintomatología depresiva (Gráfico 2). Nuestro estudio no permite obtener una conclusión clara de este hecho, que puede deberse a dos circunstancias completamente diferentes: (a) que el malestar asociado a la sintomatología depresi- 
va decrezca por efecto del tratamiento, o (b) que las personas que mantienen la intensidad de los síntomas abandonan el tratamiento. Sería preciso el diseño de estudios longitudinales para encontrar apoyo a cualquiera de las dos hipótesis.

Este mismo hecho se observa cuando utilizamos el SCL-90-R, mediante el cual observamos cómo los niveles de malestar son máximos en todas las escalas (salvo la de Ansiedad Fóbica), especialmente en la de Depresión, en tanto que los grupos más avanzados declaran progresivamente menos síntomas en todas las dimensiones, siendo el decremento más notable el que afecta a los síntomas depresivos (Gráfico 1). Esta circunstancia, que ya ha sido observada en otros estudios (Pedrero et al., 2003), puede ser explicada también por alguna de las hipótesis anteriores.

No sucede lo mismo cuando observamos las escalas sindrómicas del MCMI-II, en el que, como hemos observado, únicamente se reducen significativamente las escalas de Ansiedad y Depresión Mayor, en tanto que el resto permanecen prácticamente estables al avanzar el momento de tratamiento. La razón de este hecho puede deberse precisamente a que lo que el MCMI-II pretende medir no es sintomatología, como sucede con los otros dos cuestionarios, sino la forma en que tales síntomas se agrupan para configurar algún síndrome. La propia estructura del cuestionario liga estos cuadros a características estables de la personalidad, por lo que necesariamente nos informa de agrupaciones de síntomas que no desaparecen con tanta facilidad. Sí informa de la disminución de la severidad en síndromes depresivos y ansiosos, más sujetos a circunstancias ambientales mutables, pero tal disminución no se produce en la misma magnitud en la distimia, por ejemplo, que es, por definición, un cuadro que se caracteriza por su estabilidad temporal, y cuya diferencia con el Trastorno de Personalidad Depresiva es objeto de controversia (Hernández, Leal y Pérez, 2002; Vallejo y Vallejo, 2000).

Este hecho daría ventaja, en principio, MCMI-II, si utilizamos los cuestionarios con objetivos diagnósticos. La escala X (Sinceridad) de este cuestionario intenta reflejar la propensión del sujeto a ser reservado y ocultar síntomas, o bien a ser franco y expresarse libremente; ambos extremos anularían el test por exceso en una de las dos tendencias. De este modo, puntuaciones inferiores a 145 o superiores a 590 anularían los resultados del test. Sin embargo, en nuestra experiencia puntuaciones superiores a 590 se corresponden en una gran mayoría de los casos con sujetos con una patología muy severa, de tipo psicótico, que contestan afirmativamente a un gran número de items correspondiéndose con la experimentación de un elevado número de síntomas, que es posible confirmar en entrevistas posteriores. En efecto, por una parte la escala $X$ se alimenta de los items contestados afirmativamente, y, por otra, como puede observarse en la Tabla 2, si bien la escala $Y$ (Deseabilidad) correlaciona ligeramente, en un sentido u otro, con un buen número de escalas sindrómicas o sintomatológicas (del SCL-90-R), la escala X lo hace, con gran magnitud del efecto, con la práctica totalidad de las escalas, pero especialmente con el Pensamiento Psicótico del MCMI-II y con la Ideación Paranoide y el Psicoticismo, así como con todos los índices generales de malestar del SCL-90-R. Todo ello nos lleva a pensar que, de aplicarse la instrucción de que los tests con puntuaciones superiores a 590 en la escala $X$ deben ser anulados, el MCMI-II impediría la evaluación de los sujetos con patología más severa. Ciertamente, así se advierte en el manual del cuestionario, cuando se constata su inadecuación para evaluar dificultades ordinarias de la vida cotidiana y estilos normales de personalidad, por un lado, y patologías más graves, por otro. Sin embargo, tomar en consideración a aquellos sujetos que obtienen más de 590 en la escala X nos permite detectar la presencia de síndromes severos y estudiar su patología, y así lo hemos hecho en el presente estudio. Dificultades similares se han observado en la escala $V$, encontrándose, mediante técnicas de aleatorización de respuestas que el MCMI-III -aunque la técnica es aplicable con idénticos resultados al MCMI-II- es incapaz de detectar al $50 \%$ de los sujetos que contestan al azar (Charter y López, 2002).

Nuestro estudio nos permite explorar la sensibilidad del MCMI-II en las escalas de Abuso de Alcohol y de Drogas, en la medida en que todos los participantes comparten los criterios necesarios para estimar un diagnóstico de abuso o dependencia de su droga principal. Observamos en la Tabla 5 cómo la sensibilidad de la escala de Abuso de Alcohol sólo alcanza el $61 \%$, mientras que la de Abuso de Drogas no alcanza el 50\% para los consumidores de heroína y cocaína, y ni siquiera el $20 \%$ para los consumidores de cannabis. Datos similares son obtenidos en otros estudios con la segunda (Van Hoek, 1995) y tercera versión de este cuestionario (Craig, 1997).

Una razón para esta cuestionable utilidad diagnóstica de los cuestionarios estudiados se debe a la propia confección de las escalas. Gottlib y Cane (1989) estudiaban los items que componían las escalas de estos tres y otros cuestionarios, encontrando que, ateniéndose a la descripción de las categorías diagnósticas, de los 21 items del BDI 12 medían realmente síntomas propios de depresión, 4 eran comunes a ansiedad y depresión, 1 era propio y exclusivo de la ansiedad y 4 no estaban relacionados con ninguno de los dos constructos. La escala de Depresión del SCL-90 presentaba 8 items propios, 1 común, 1 de ansiedad y 3 no relacionados. El MCMI presentaba 11 items propios, 6 comunes, 2 de ansiedad y hasta 17 ítems no relacionados con ninguno de los dos constructos. Bien es cierto que la versión del MCMI que estudiaba era la primera, pero hay que hacer constar que en el propio manual de 
la segunda versión (Millon, 1999, p.72) se explica que ambas escalas -Distimia y Depresión Mayor- apenas han visto modificados sus items, y sólo en la segunda se ha alterado sustancialmente la ponderación de algunos items prototípicos, por lo que los resultados de este estudio son aplicables a la nueva versión. Estas diferencias en la distribución de los items explican las correlaciones entre las diversas escalas, que se muestran en la Tabla 7: es muy elevada entre BDI y SCL90-R-Depresión, y sensiblemente menores cuando se comparan estas dos con las del MCMI-II.

Lo cual nos lleva a otra cuestión de interés en el estudio de estos cuestionarios, la que se refiere a su validez convergente y discriminante. Los estudios previos son consistentes en atribuir adecuados niveles de convergencia entre las medidas de estos tests, pero pobres resultados cuando se evalúa su capacidad discriminante, y ello cuando nos referimos al BDI (Steer, y Clark, 1997), al SCL-90-R (Brophy et al., 1988) o al MCMI, tanto en las escalas del Eje I (McCann, 1990) como en las del Eje II (Rossi, Van den Brande, Tobac, Sloore y Hauben, 2003; Widiger y Sanderson, 1987). Como veíamos en la Tabla 7, la convergencia entre las puntuaciones de las diversas escalas de depresión es muy fuerte, especialmente en el caso del BDI y la escala de Depresión del SCL-90-R. Sin embargo, en la Tabla 6 observamos que también lo es entre éstas y casi todas las demás. El BDI correlaciona 0,80 con la Depresión del SCL-90-R, pero lo hace entre 0,51 y 0,71 con todas las demás; especialmente sugestivo es el hecho de que la covariación con el Índice General Sintomático es el mismo que con la Escala de Depresión $(0,79)$. Por otra parte, este IGS se predice a partir de la escala de Ansiedad o la de Depresión por separado por encima del 80\%, y ambas juntas predicen más del $90 \%$ de su varianza. Lo que viene a ratificar la idea, propuesta por diversos estudios (Brophy et al., 1988; Cyr, McKenna-Foley y Peacock, 1985) de que el SCL-90-R (y acaso también el BDI, como sugiere la observación de la Tabla 6) sólo es capaz de medir una única dimensión de malestar general (global distress), confirmado mediante análisis factoriales que encuentran consistentemente un único factor, no rotado, que explica la mayor parte de la varianza del test. Probablemente, esta dimensión de malestar general se nutra principalmente de los síntomas ansioso/depresivos, que serían mejor conceptualizados como correlatos de una situación de estrés que de una entidad mórbida estable, y que serían mejor explicados a partir de modelos procesuales psicobiológicos que mediante descripciones categoriales (Pedrero et al., 2003).

Desde esta perspectiva, la utilidad diagnóstica de estos instrumentos es muy limitada, en tanto que ofrecen poco más que una descripción más o menos exhaustiva de síntomas inespecíficos que denotan más bien un estado transitorio de estrés que una auténtica psicopatología estable. La derivación de diagnósticos a partir de los datos proporcionados por estos cuestionarios, incluso los obtenidos a partir de otros métodos como entrevistas estructuradas, cuando se realizan en los primeros momentos de un proceso terapéutico que se inicia con la demanda de ayuda, no pasan de ser una "foto fija" con mínima capacidad explicativa sobre el proceso general, y su uso como instrumentos de estimación de cambio puede ser, sin duda, una labor "agradecida" en la medida en que una gran parte de los síntomas van a desaparecer en los primeros momentos de la intervención o, cuando menos, van a reducir significativamente su intensidad. Lo cual es un dilema difícil de asimilar por un procedimiento que prioriza la simplificación que supone tomar como "libros de cabecera" las clasificaciones diagnósticas, cada vez más empeñadas en presentar los trastornos psicopatológicos como aquellos que se definen por una serie de descripciones (demasiado constreñidas), perdiendo de vista los orígenes de la psicopatología y la riqueza que ésta encierra (Espada, 1997).

Parece claro que, cuando el propósito de la evaluación es la aproximación diagnóstica, debemos decantarnos por instrumentos como el MCMI-II, que trascienden de la mera exploración de síntomas subjetivos, dando un paso más hasta proponer que tal configuración de síntomas, en base al conocimiento acumulado, comportan, con cierto grado de probabilidad, la presencia de un síndrome y, acaso, de un trastorno. Los resultados mostrados en la Tabla 10 parecen apoyar la estructura de las escalas de síndromes clínicos del cuestionario de Millon: los más determinados por la sintomatología recogida por el SCL-90-R son, precisamente, los depresivos (Distimia y Depresión Mayor) y el de Ansiedad, pero entre ambos hay alguna diferencia de interés: mientras la ansiedad tiene un componente más somático, la distimia tiene un componente más cognitivo, asociado al psicoticismo, y la depresión mayor es determinada además por pensamientos y conductas sugestivas de obsesión y compulsión. Los síntomas depresivos participarían de los tres síndromes, pero en una gradación desde la menor determinación en el caso de la ansiedad hasta la máxima en la depresión mayor. El resto de síndromes parece responder a una configuración sintomatológica razonablemente ajustada a la conceptualización en la que se sustentan. Como apreciamos también en la Tabla 8, los sujetos que superan el punto de corte para la estimación de presencia de un trastorno distímico presentan cifras medias notablemente elevadas en la escala de Depresión del SCL-90-R y también en el BDI; sin embargo, quienes serían acreedores a un diagnóstico de trastorno depresivo mayor no difieren prácticamente en nada de aquéllos: declaran niveles similares de síntomas depresivos, luego sus diferencias deben atribuirse a otros componentes. Observamos que la escala de Ansiedad del SCL-90-R parece estar más relacionada con los síndromes depresivos que con el de ansiedad: 
la puntuación es más elevada en los sujetos que presentan Distimia (según los puntos de corte de TB=75), aún más en los de Depresión Mayor, y sensiblemente menor en los que parecen presentar un síndrome de Ansiedad. Como veíamos en la Tabla 10, esa ansiedad de la depresión mayor parecería estar más en relación con pensamientos e impulsos indeseados, absurdos, difíciles de resistir, evitar o eliminar, que provocan angustia, y las conductas consecuentes encaminadas a la evitación o el escape de tales ideas.

Por el contrario, los autoinformes de síntomas parecen más adecuados a la relación paciente-terapeuta, en la medida en que nos informan de la experiencia subjetiva de malestar de forma directa y personalizada. Lo cual supone, sin duda, la base sobre la que se sustenta el proceso de ayuda demandado por el paciente y provisto por el profesional. La reducción de los síntomas desde los primeros momentos de la intervención supone, además, una fuente de reforzamiento de innegable utilidad para favorecer la adherencia y el cumplimiento del tratamiento, en la medida en que las atribuciones causales del cambio por parte del paciente apunten a factores internos (capacidades y esfuerzo) e intermedios (cumplimiento de pautas del tratamiento, tanto farmacológico como psicoterapéutico) y no a factores internos o externos incontrolables. La asignación de diagnósticos es de incuestionable interés para la comunicación entre profesionales de diversas disciplinas, así como para las tareas propias de la investigación, pero puede interferir en la consecución de objetivos terapéuticos en el punto en que puedan ser interpretadas por cualquiera de las partes como "etiquetas" o condiciones más o menos inmutables o incontrolables.

Por ello, consideramos la conveniencia de combinar ambos métodos: las ventajas del MCMI-II son incuestionables, en cuanto al volumen de información que nos proporciona sobre los dos primeros ejes clasificatorios; presenta una apreciable especificidad y puede orientar la evaluación hacia diagnósticos complejos a partir de un pequeño gasto de tiempo y esfuerzo (175 items, tiempo medio 20-40 minutos). Pero el uso de una escala de síntomas puede favorecer la conexión paciente-terapeuta en la medida en que el trabajo mutuo se establece sobre vivencias reales y no sobre constructos clasificatorios. En este punto, no parece que el SCL-90-R proporcione mucha más información de la que ofrece el BDI, con 69 items menos y similar dificultad de cumplimentación. Cuando se requiere una batería de tests para explorar diversas dimensiones (no todas ligadas a la psicopatología) parece adecuado restringir las demandas planteadas al paciente, respetando los principios de parsimonia hasta donde sea posible. La exhaustividad en la evaluación debe contar necesariamente con el complemento de entrevistas personalizadas que den sentido a la información obtenida mediante los cuestionarios y facilite el establecimiento de objetivos de tratamiento.

\section{REFERENCIAS}

Aben, I., Verhey, F., Lousberg, R., Lodder, J. y Honig, A. (2002). Validity of the Beck Depression Inventory, Hospital Anxiety and Depression Scale, SCL-90, and Hamilton Depression Rating Scale as screening instruments for depression in stroke patients, Psychosomatics, 5, 38693.

Anda, R.F., Williamson, D.F., Escobedo, L.G., Mast, E.E., Giovino, G.A. y Remington, P.L. (1990). Depression and the dynamics of smoking: A national perspective, Journal of the American Medical Association, 264, 1541-1545.

Arias Horcajadas, F. (2001). Comorbilidad de trastornos psiquiátricos y trastornos adictivos, en E. Ochoa Mangado (Dir.), Antagonistas opiáceos en las dependencias. Clínica de Naltrexona. (pp. 105-125). Madrid: Ars Medica.

Arias, F., López Ibor, J.J. y Ochoa, E. (1997). Comorbilidad psiquiátrica en dependientes de opiáceos en tratamiento con naltrexona, Adicciones, 2, 235-253.

Beck, A.T. (1976). Cognitive therapy and the emotional disorders. New York: International University Press.

Beck, A.T., Rush, A.J., Shaw, B.F. y Emery, G. (1979). Cognitive therapy of depression. New York: Guilford Press.

Beck, A.T., Ward, C.H., Mendelson, M., Mock, J. y Erbaugh, J. (1961). An inventory for measuring depression, Archives of General Psychiatry, 4, 561-571.

Breslau, N., Kilbey, M.M. y Andresky, P. (1993). Nicotine dependence and major depression, Archives of General Psychiatry, 50, 31-35.

Brophy, C.J., Norvell, N.K. y Kiluk, D.J. (1988). An examination of the factor structure and convergent and discriminant validity of the SCL-90R in an outpatient clinic population, Journal of Personality Assessment, 52, 334-340.

Casas, M. y Guardia, J. (2002). Patología psiquiátrica asociada al alcoholismo, Adicciones, 14(Supl. 1), 195-219.

Charter, R.A. y López, M.N. (2002). Millon Clinical Multiaxial Inventory (MCMI-III): the inability of validity conditions to detect random responders, Journal of Clinical Psychology, 12, 1615-1617.

Conde, V., Esteban T, y Useros, E. (1976). Revisión crítica de la adaptación castellana del Cuestionario de Beck. Revista de Psicología General y Aplicada, 31, 469-497.

Craig, R.J. (1997). Sensitivity of MCMI-III scales T (drugs) and $B$ (alcohol) in detecting substance abuse. Substance Use and Misuse, 10, 1385-1393.

Cyr, J.J., McKenna-Foley, M.M. y Peacock, E. (1985). Factor structure of the SCL-90R: is there one?. Journal of Personality Assessment, 49, 571-578.

Derogatis, L. (1975). Brief symptom inventory. Baltimore: Clinical Psychometric Research.

Derogatis, L. (1983). SCL-90-R. Administration, scoring and procedures manual. Baltimore: Clinical Psychometric Research. 
Derogatis, L.R., Lipman, R.S. y Covi, L. (1973). SCL-90: An outpatient psychiatric rating scale. Preliminary report. Psychopharmacol Bull, 9, 13-28.

Derogatis, L.R. (2002). SCL-90-R. Cuestionario de 90 síntomas. Manual. Madrid: Tea Ediciones.

Espada Largo, F.J. (1997). Relaciones entre Ansiedad y Depresión. Psicología.com, Revista Electrónica de Psicología, 1. [online]. Recuperado el 28 de agosto de 2004, de http://www.psiquiatria.com/psicologia/ vol1num1/artic_3.htm

Franken, I.H.A. y Hendricks, V.M. (2001). Screening and Diagnosis of Anxiety and Mood Disorders in Substance Abuse Patients. American Journal on Addictions, 1, 3039.

Gawin, F. y Kleber, R.H. (1986). Abstinence symptomatology and psychiatric diagnosis in cocaine abusers: clinical observations. Archives of General Psychiatry, 43, 107113.

Gotlib, I.H. y Cane, D.B. (1989). Self-Report assessment of depression and anxiety. En P. C. Kendall y Watson (Eds), Anxiety and depression: Distinctive and overlapping features (pp. 131-169). New York: Academic Press.

Hernández Viadel, M., Leal Cercos, C. y Pérez Prieto, J.F. (2002). Trastorno depresivo de la personalidad. Psiquiatría Biológica, 5, 200-206.

Hesselbrock, M.N., Hesselbrock, V.M., Tennen, H., Meyer, R.E. y Workman, K.L. (1983). Methodological considerations in the assessment of depression in alcoholics. Journal of Consulting and Clinical Psychology, 51, 399-405.

Kidorf, M., Disney, E.M., King, V.L., Neufeld, K., Beilenson, P.L. y Brooner, R.K. (2004). Prevalence of psychiatric and substance use disorders in opioid abusers in a community syringe exchange program. Drug and Alcohol Dependence, 2, 115-122.

Marlowe, D.B. y Wetzler, S. (1994). Contributions of discriminant analysis to differential diagnosis by selfreport. Journal of Personality Assessment, 2, 320-331.

Martínez Higueras, I.M. (1993). Estudio sintomático de drogodependientes en tratamiento con el SCL-90. Psiquis, 4, 152-161.

McCann, J.T. (1990). A multitrait-multimethod analysis of the MCMI-II Clinical Syndrome Scales. Journal of Personality Assessment, 3/4, 465-476.

Meyer, R.E. (1986). How to understand the relationship between psychopathology and addictive disorders: another example of the chicken and the eggs. En R.E.Meyer (Ed), Psychopathology and addictive disorders (pp. 3-16). New York: Guilford Press.

Miller, N.S., Klamen, D., Hoffman, N.G. y Flaherty, J.A. (1996). Prevalence of depression and alcohol and other drug dependence in addictions treatment populations. Journal of Psychoactive Drugs, 2, 111-124.

Millon, T. (1987). Manual for the Millon clinical multiaxial inventory - Second edition (MCMI-II). Minneapolis: National Computer Systems.

Millon, T. (1999). MCMI-II. Inventario clínico multiaxial de Millon-II. Manual. Adaptación española de A. Ávila-
Espada (Dir.), F. Jiménez Gómez (Coord.) y cols. Madrid: Tea Ediciones.

Moffet t, L.A. y Radenhausen, R.A. (1990). Assessing depression in substance abusers: Beck Depression Inventory and SCL-90R. Addictive Behaviors, 15, 179181

Morgan, MJ. (2000). Ecstasy (MDMA): a review of its possible persistent psychological effects. Psychopharmacology,152, 230-248.

Ochoa Mangado, E. (2000). Cocaína y comorbilidad psiquiátrica. Actas Españolas de Psiquiatría, 1, 40-52.

Ortiz Cobo, A. (1998). Epidemiología y modelos explicativos del trastorno dual. En J.Cabrera Forneiro (Ed.), Patología Dual (pp. 45-59). Madrid: Agencia Antidroga.

Parloff, M.B., Kelman, H.C. y Frank, J.D. (1954). Comfort, effectiveness, and self-awareness as criteria for improvement in psychotherapy. American Journal of Psychiatry, 3, 343-51.

Pedrero Pérez, E.J., Puerta García, C, Lagares Roibas, A. y Sáez Maldonado, A. (2003). Prevalencia e intensidad de trastornos de la personalidad en adictos a sustancias en tratamiento en un Centro de Atención a las Drogodependencias. Trastornos Adictivos, 3, 241-255.

Pérez de los Cobos, J. y Casas, M. (1993). Dependencia de opiáceos y psicopatología concomitante, en J.Cadafalch y M.Casas (Eds.), El paciente heroinómano en el Hospital General (pp. 149-168). San Sebastián: Citrán.

Regier, D.A., Farmer, M.E., Rae, D.S., Locke, B.Z., Keith, S.J. Judd, L.L., et al. (1990). Comorbidity of mental disorders with alcohol and other drug abuse. Results from the Epidemiologic Catchment Area (ECA) Study. JAMA, 19, 2511-2518.

Retzlaff, P. (1996). MCMI-III Diagnostic Validity: Bad Test or Bad Validity Study. Journal of Personality Assessment, 2, 431-437.

Richter, P., Werner, J., Heerlein, A., Kraus, A. y Sauer, H. (1989). On the validity of the Beck Depression Inventory. A review. Psychopathology, 3, 160-168.

Rossi, G., Van den Brande, I., Tobac, A., Sloore, H. y Hauben, C. (2003). Convergent Validity of the MCMIII Personality Disorder Scales and the MMPI-2 Scales. Journal of Personality Disorders, 4, 330-340.

Rubio Valladolid, G. (1998). Métodos diagnósticos de trastornos psiquiátricos en poblaciones con abuso de drogas. En J.Cabrera Forneiro (Ed). Patología Dual (1732). Madrid: Agencia Antidroga.

Rubio, G., Bango, J., Fadón, P., Pascual, J. y Santo-Domingo, J. (1998). Trastornos psiquiátricos y consumo de MDMA. En G.Rubio y G. Álamo (Eds), Éxtasis: una droga para la controversia (pp. 69-88). Valencia: Promolibro.

San, L. (2004). Consenso de la SEP sobre patología dual (4). Barcelona: Psiquiatría Editores.

Sánchez-Hervás, E., Tomás Gradolí, V. y Morales Gallús, E. (2001) Evaluación psicopatológica en dependientes al alcohol, heroína y cocaína mediante el Brief Symptom Inventory. Adicciones, 1, 61-66.

Sanz, J, Navarro, M. E. y Vázquez, C. (2003) Adaptación española del Inventario para la Depresión de Beck - II (BDI-II): 1. Propiedades psicométricas en estudiantes 
universitarios. Análisis y Modificación de Conducta, 29, 239-288.

Sanz, J., Perdigón, A. L. y Vázquez Valverde, C. (2003) Adaptación española del Inventario para la Depresión de Beck-II (BDI-II): 2. Propiedades psicométricas en población general. Clínica y Salud, 3, 249-280.

Sanz, J. y Vázquez, C. (1998). Fiabilidad, validez y datos normativos del inventario para la depresión de Beck. Psicothema, 2, 303-318.

Soler Insa, P.A., Gascón Barrachina, J. y Grau Morillo, M. (1982). Síndrome depresivo y alcoholismo. En M. CASAS (Coord). Trastornos psíquicos en toxicomanías (pp. 239-248). Barcelona: Ediciones Neurociencias.

Steer, R.A. y Clark, D.A. (1997). Psychometric Characteristics of the Beck Depression Inventory-II with College Students. Measurement and Evaluation in Counseling and Development, 3, 128-136.

Strauman, T.J. y Wetzler, S. (1992). The factor structure of SCL-90 and MCMI scale scores: within-mesure an interbattery analyses. Multivariate Behavioral Research, $1,1-20$.

Vallejo, J. y Vallejo, G. 2000, Trastornos de personalidad y depresión. Referencia concreta a la distimia. Psiquiatría Biológica, 7, 160-167.
Van Hoek, B. (1995) Use of a structured interview to evaluate the validity of the Alcohol and Drug Dependence Scales of the Millon Clinical Multiaxial Inventory II, Dissertation Abstracts International: Section B: The Sciences $y$ Engineering, 56(5-B), 2935.

Vázquez, C. 1995, Evaluación de los trastornos depresivos y bipolares. En A. Roa (Ed), Evaluación en Psicología Clínica y de la Salud (pp. 163-209). Madrid: CEPE.

Weiss, R.D., Griffin, M. L. y Mirin, S.M. (1989). Diagnosing major depression in cocaine abusers: The use of depression rating scales. Psychiatry Research, 3, 335343.

Wetzler, S., Kahn, R., Strauman, T.J. y Dubro, A. (1989). Diagnosis of major depression by self report. Journal of Personality Assessment, 1, 22-30.

Widiger, T.A. y Sanderson, C. (1987). The convergent and discriminant validity of the MCMI as a measure of the DSM-III Personality Disorders. Journal of Personality Assessment , 2, 228-242.

Ziedonis, D. M. (1992). Comorbid psychopathology and cocaine addiction, En T. R. Kosten y H. D. Kleber, Clinician's guide to cocaine addiction (pp. 335-358), New York: The Guildford Press. 